\title{
Lingual Frenulum Phenotypes in Brazilian Infants With Congenital Zika Syndrome
}

The Cleft Palate-Craniofacial Journal I-8 (C) The Author(s) 2018 Reprints and permission: sagepub.com/journalsPermissions.nav DOI: 10.1 I77// 0556656/8766999 journals.sagepub.com/home/cpc (S)AGE

\author{
Cristiane Sá Roriz Fonteles, DDS, MS, PhD', \\ Erlane Marques Ribeiro, MD, MS, $\mathrm{PhD}^{2,3}$, \\ Marinisi Sales Aragão Santos, SLPIAud', \\ Rebeka Ferreira Pequeno Leite, SLP/Aud ${ }^{2}$, Gabryela Sales Assunção, SLP/Aud ${ }^{2}$, \\ André Jalles Monteiro, MS, PhD', André Luiz Santos Pessoa, MD', \\ Célia Maria Giacheti, SLP/Aud, PhD $^{4}$, Sáile Cavalcante Kerbage, MD², \\ Thyciana Rodrigues Ribeiro, DDS, MS, PhD', and \\ Luciano Pamplona de Góes Cavalcanti, BS, MS, PhD 5
}

\begin{abstract}
Objective: The present study aimed to evaluate lingual frenulum in children affected by congenital Zika syndrome (CZS) and to analyze the association of lingual frenulum phenotypes with other variables.

Design: This present work had a cross-sectional, descriptive study design.

Setting: This study was carried out in Fortaleza (Brazil). The health professionals provided tertiary level of care. Data collection occurred during a multidisciplinary task force for evaluating infants affected by CZS in December 2 to 3, 2016.

Patients: Fifty-four patients with CZS (I-12 months old; 32 girls and 22 boys) were recruited from a population of 70 infants. Interventions: A multidisciplinary group comprised of speech-language pathologist/audiologists and pediatric dentists evaluated all patients through an intraoral examination and a specific tongue maneuver protocol for infants.

Main Outcome Measures: Lingual frenulum visibility was the primary outcome measure. Before initiating the study, we hypothesized that children with CZS had an absent lingual frenulum.

Results: Lingual frenula were visible in $34(63 \%)$ infants, whereas in $20(37 \%)$ infants lingual frenula visibility required a specific maneuver to retract the tongue. Six of 20 infants presented posteriorly positioned lingual frenula that were visible after maneuver. Lingual frenula were covered by mucous tissue in 14 infants. Presence of posterior frenulum was associated with dysphagia $(P=$ 0.038). However, the presence of dysphagia in a multivariate model did not associate with the presence of a posterior lingual frenulum $(P=.069)$ or neurologic symptoms $(P=.056)$.

Conclusion: Children with CZS showed predominance of a posterior lingual frenula covered by an overlapping curtain-like mucous membrane.
\end{abstract}

\section{Keywords}

microcephaly, Zika virus, lingual frenum, stomatognathic system

\footnotetext{
' Department of Clinical Dentistry, Federal University of Ceará (UFC), Fortaleza, Ceará, Brazil

${ }^{2}$ Albert Sabin Children's Hospital (HIAS), Fortaleza, Ceará, Brazil

${ }^{3}$ Faculty of Medicine, Christus University Center (UNICHRISTUS), Fortaleza, Ceará, Brazil

${ }^{4}$ Department of Speech-Language Pathology and Audiology, São Paulo State University Júlio de Mesquita Filho (UNESP), Marília, São Paulo, Brazil

${ }^{5}$ Department of Community Health, Federal University of Ceará (UFC), Fortaleza, Ceará, Brazil
}

\section{Corresponding Author:}

Cristiane Sá Roriz Fonteles, DDS, MS, PhD, Department of Clinical Dentistry, Federal University of Ceará (UFC), Rua Monsenhor Furtado, I273, Rodolfo Teófilo, Fortaleza, Ceará 60430, Brazil.

Email: cfontele@ufc.br 


\section{Introduction}

An increase in the number of children born with microcephaly in Northeast Brazil by mid-2015 (Campos et al., 2015; Heukelbach et al., 2016) led to the establishment of a new pattern of birth defects, called congenital Zika syndrome (CZS) (Schuler-Faccini et al., 2016; Cavalcanti et al., 2017; Del Campo et al., 2017). In 2016, Moore et al. (2017) acknowledged that although children with congenital Zika infection expressed features that were common to other congenital infections, 5 specific characteristics distinguished this novel syndrome as a single entity: (1) severe microcephaly with partially collapsed cranium, (2) reduced cerebral cortices with specific pattern of brain injury and associated subcortical calcifications, (3) posterior ocular findings including macular scarring and focal pigmentary retinal mottling, (4) congenital contractures involving one or multiple joints (ie, arthrogryposis, clubfoot), and (5) pronounced hypertonia soon after birth, with extrapyramidal symptoms.

Despite the current knowledge on CZS and its neurologic repercussions (Schuler-Faccini et al., 2016; Van der Linden et al., 2016; Cavalcanti et al., 2017), little is known on the intraoral structural and functional aspects of this syndrome and the influence of such phenotype on the overall ability of these children to suction, swallow, feed, and ultimately thrive (de Fatima Vasco Aragao et al., 2016; Ventura et al., 2016). Previously, Del Campo et al. (2016) published a study based on the result of a multidisciplinary evaluation and neuroimaging of 83 infants expressing findings consistent with CZS. While providing an overview of this phenotypic spectrum, the authors reported absence of lingual frenulum in this population. By definition, the lingual frenulum consists of a thin fold of mucosal attachment located at the midline and extending from the ventral surface of the tongue to the floor of the mouth with the main role of providing stability to the tongue (Fehrenbach and Herring, 2002; Mintz et al., 2005). Hence, tongue mobility can be influenced by the thickness of the lingual frenulum and the level of attachment of this membrane to the tongue and floor of the mouth. Tissue boundaries and texture are determined during the embryonic period (Martinelli et al., 2013), and the phenotypic spectrum that arises may potentially influence function by limiting or not tongue mobility (Knox, 2010).

The present study aimed to evaluate the anatomy of the lingual frenulum of a group of children previously diagnosed with CZS in order to test the hypothesis that children with CZS express absence of lingual frenulum. We have also analyzed the association of lingual frenulum phenotypes (visible, posteriorly positioned, submucous, or absent) with other related variables, such as presence of microcephaly, neurologic symptoms, and dysphagia.

\section{Materials and Methods}

\section{Ethical Aspects}

This study is part of a larger multidisciplinary research project designed to prospectively monitor cases of embryopathy

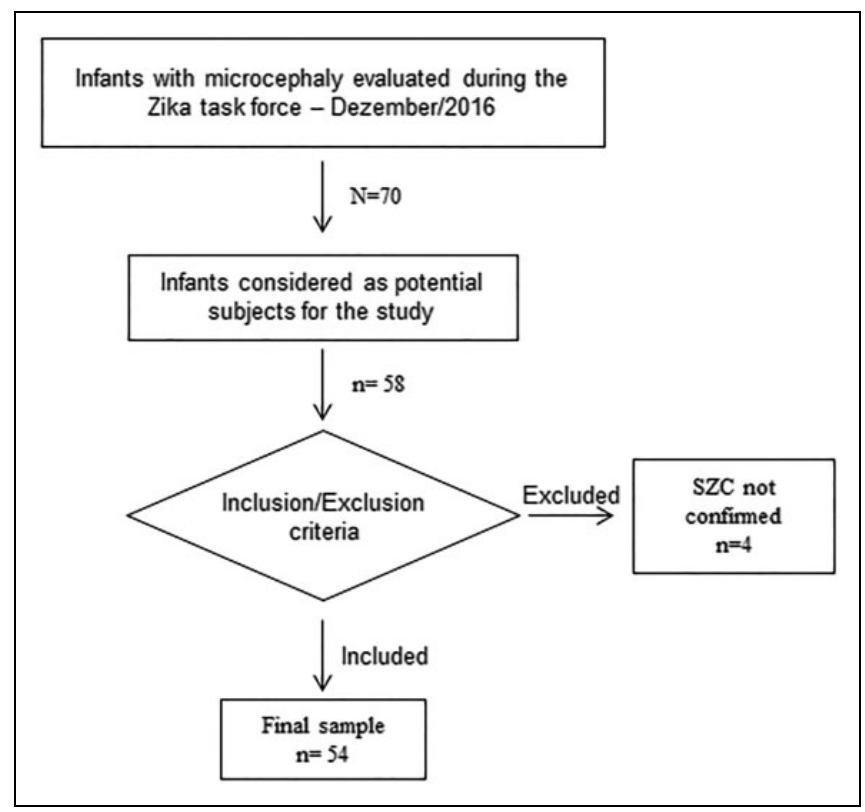

Figure I. Flowchart showing patient recruitment and final sample size.

caused by Zika virus infection in the state of Ceará and was approved by the Ethics Committee in Research of the Albert Sabin Children's Hospital (CAAE: 57109816.7.0000.5042), through protocol number 1.743.023. Parents or legal guardians of all children signed informed consent forms prior to the child's enrollment in the study.

\section{Study Design, Location, and Population}

This work had a cross-sectional, descriptive study design. Children diagnosed with CZS were referred to participate in the study by reference centers for the treatment of CZS located in different parts of the state of Ceará, in northeast Brazil. Data collection occurred during a multidisciplinary task force provided by a team of health professionals in December 2 to 3 , 2016. These multidisciplinary evaluations occur every 6 months for the prospective evaluation of children diagnosed with CZS, at the Center for Perfecting Sight See Hope Revive (CAVIVER, Fortaleza, Ceará, Brazil), a nonprofit health organization. Intraoral examination and assessment of the lingual frenulum (insertion and anatomy) were carried out on the same day by 1 pediatric dentist and 2 speech-language pathologists/ audiologists. This study incorporated data previously collected by the multidisciplinary team on the neurologic evaluation of these patients and assessment of swallowing disorders (dysphagia).

\section{Patient Enrollment Criteria and Study Sample}

Children of both sexes born in the state of Ceará and diagnosed with CZS were recruited to participate in this study (Figure 1). The final study population consisted of 54 infants (32 females and 22 males), from 1 to 12 months of age (median age: 9 months). The diagnosis of CZS was based on the 
physical examination of these patients and neuroimaging findings provided by professionals previously trained on the diagnosis of this syndrome, following criteria established by Moore et al. (2017).

\section{Data Collection}

Data collection included standardized anatomical and functional evaluations performed by a multidisciplinary team comprised of speech-language pathologist/audiologist and a pediatric dentist. Intraoral photographs to register growth and development were obtained from all infants. Images were analyzed by 2 professionals ( 1 pediatric dentist and 1 speechlanguage pathologist/audiologist) to confirm clinical evaluation, and when lack of consensus was an issue, a third examiner was consulted. As part of the CZS protocol, infants had full clinical and neurological evaluations by a multidisciplinary team and were examined for the presence of dysphagia. Data concerning dysphagia, neurologic assessment, presence of microcephaly at birth, and body weight for age were collected from patients' charts and used for further analysis.

For this study, dysphagia was categorized as being present or absent. In case presence of dysphagia was identified in patients" chart, level of dysphagia was registered as "1" or mild - delayed, slow control, and transport of the food bolus, without signs of laryngeal penetration upon auscultation; " 2 " or moderate - same pattern as mild dysphagia, but with signs of laryngeal penetration and risk of aspiration upon auscultation; " 3 " or severe - characterized by the presence of substantial aspiration with signs of respiratory alteration and absence or failure of complete deglutition of the food bolus, compromising oral feeding. Laryngeal penetration was defined as passage of the food bolus into the larynx, but not through the vocal folds, while aspiration was defined as passage of the food bolus through the vocal folds.

Once gathered from patients' charts, neurologic data were categorized and subdivided as signs of pyramidal tract lesion (weakness, hyperreflexia, tremors, spastic hypertonia) and signs of extrapyramidal tract lesion (mostly dystonia, or loss of synchronicity of movements). The presence of microcephaly at birth and low body weight for age were calculated based on the $z$-scores for these variables.

\section{Pediatric Intraoral Examination}

A detailed intraoral examination to evaluate the infants' stomatognathic system was performed by a single experienced pediatric dentist. During intraoral examination, one of the parents was comfortably seated on a reclining chair with the infant on their lap. The position was adjusted as needed. Newborns and edentulous infants were examined in the arms of one of their parents. In the presence of teeth, infants were examined by instructing the parent to sit on a chair facing the dentist, who was seated in the operator chair knee-to-knee with the parent. The infant was positioned on the parent's lap facing the parent, with 1 leg wrapped around each side of the parent, while the child's head was lowered on to the dentist's lap for the examination (knee-to-knee technique). Artificial light was used for intraoral illumination. Soft tissue examination and dental evaluation were performed, followed by the evaluation of labial and lingual frenula (anatomic aspects and level of insertion). For the purpose of the present study, results of the pediatric dental examination were restricted to findings related to the lingual frenulum and surrounding tissues.

\section{Myofunctional Evaluation}

Speech-language pathologists/audiologists evaluated the following aspects: global muscular tone, lip tone, tongue and cheeks, global posture, postural control, and cervical spine control. The lingual frenulum was evaluated by observing the following aspects: frenulum visibility, thickness, and level of frenulum attachment to the tongue and floor of mouth, according to the protocol established by Martinelli et al. (2013), adapted to be applied with the infant at rest. During tongue elevation, the presence/absence of lingual frenulum visibility was assessed. In the presence of a visible lingual frenulum, the thickness of the frenulum was determined (thin or thick), followed by the level of attachment of the lingual frenulum (1) to the ventral side of the tongue - the mid-third, between the mid-third and the apex, or at the apex, and (2) to the floor of the mouth - if attachment to this area was visible at the level of the sublingual caruncle (right and left submandibular ducts) or at the level of the inferior alveolar crest. In case the frenulum did not become visible following a simple elevation maneuver (simple maneuver), the tongue was once more elevated and retracted (specific maneuver) as a final attempt to visualize the lingual frenulum (Marchesan et al., 2014). In case visibility was not possible following both maneuvers, the lingual frenulum was considered to be absent and information on the level of attachment and thickness (frenulum anatomy) was not registered (Marchesan et al., 2014). Thus, lingual frenulum was classified as being (1) visible - mucosal tissue band/attachment visualized through a simple elevation maneuver of the tongue, (2) posteriorly positioned-thick band or column of tissue that is located against and protrudes from the base of the tongue, visualized through a specific maneuver of the tongue, (3) submucous-discrete band or column of tissue that is located against and protrudes from the base of the tongue, covered by an overlapping curtain-like mucous membrane, visualized through a specific maneuver of the tongue (Martinelli et al., 2013), or (4) absent-total absence of tissue protruding from the base of the tongue following application of specific maneuver.

\section{Data Analysis}

The qualitative or dichotomous variables were described as absolute measures and respective percentages. Continuous variables were described by using measures of central tendencies. The association between categorical variables, such as dysphagia (presence/absence), type of lingual frenulum, 
neurologic signs, presence of microcephaly at birth, and body weight for age was tested by $\chi^{2}$ or Fisher exact tests, when appropriate. A binomial logistic regression model was built to determine the effects of presence of a posterior lingual frenulum, pyramidal/extrapyramidal signs, cervical spine control, postural control, and global muscular tone (independent variables) on the likelihood that infants would present dysphagia (dependent variable). The level of significance was set at $P \leq$ .05 . The descriptive analysis was performed by using Epiinfo software, version 3.5.4, and SPSS software, version 20.0 was used for association analysis and for the binomial logistic regression analysis.

\section{Results}

\section{Muscle Tone and Posture}

Fifty-four (32 females-59.2\%; 22 males-40.8\%) 1- to 12 month-old infants were evaluated (median age: 9 months) in the present study. Clinical evaluation revealed that $75.9 \%$ of infants presented global hypertonia, $81.5 \%$ expressed posture disorganization, and $68.5 \%$ showed inadequate cervical spine control. Low muscle tone of lips, cheeks, and tongue were also observed in $13 \%, 16.7 \%$ and $13 \%$ of patients, respectively (Table 1).

\section{Lingual Frenulum}

The lingual frenulum was observed in all infants, when simple or specific maneuvers were performed (Figure 2). Simple elevation maneuver of the tongue allowed visibility of the lingual frenulum in $34(63 \%)$ infants. However, 20 (37\%) infants required elevation followed by posterior maneuver of the tongue to adequately visualize the lingual frenulum (Figure 3). Of these 20 infants, $6(11.1 \%)$ showed a posteriorly positioned lingual frenulum (with visible level of attachment and thickness), whereas in 14 (25.9\%) infants, the lingual frenulum was identified as being submucous.

\section{Lingual Frenulum and Other Variables}

A total of 40 of 54 children were evaluated for dysphagia (not present $=10$, mild $=22$, moderate $=7$, severe $=1$ ). Of 37 patients who went through neurologic assessment, 17 presented only pyramidal signs, while 20 presented pyramidal and extrapyramidal signs. Presence of a posterior lingual frenulum did not significantly associate with the presence of microcephaly at birth $\left(\chi^{2}(1)=1.41, P=.234\right)$ or body weight for age $\left(\chi^{2}(1)=\right.$ $.98, P=.321)$. However, the presence of dysphagia showed a significant association with the presence of the pyramidal/ extrapyramidal signs $\left(\chi^{2}(1)=4.23, P=.040\right)$ and the presence of a posterior lingual frenulum $\left(\chi^{2}(1)=4.30, P=.038\right.$; Table 2 ). The presence of the pyramidal/extrapyramidal signs was not significantly associated with the presence of posterior lingual frenulum $\left(\chi^{2}(1)=0.05, P=.942\right)$. None of the 5 predictor variables were statistically significant $(P>.05)$ in the binomial logistic regression model (Table 3).
Table I. Clinical Features of Children With Congenital Zika Syndrome (CZS). ${ }^{\mathrm{a}}$

\begin{tabular}{lc}
\hline Variables & Number of Infants \\
\hline Sex & 32 \\
Female & 22 \\
Male & \\
Global muscle tonus & 41 \\
Hypertonia & 7 \\
Hypotonia & 6 \\
$\quad$ Normal & \\
Global posture & 44 \\
Disorganized & 10 \\
Organized & \\
Cervical spine Control & 17 \\
Adequate & 37 \\
Inadequate & \\
Postural control & 7 \\
Adequate & 7 \\
Inadequate & \\
Cheeks & \\
Altered & 9 \\
Normal & 45 \\
Lips & \\
Altered & 7 \\
Normal & 47 \\
Tongue & \\
Altered & \\
Normal & \\
\hline
\end{tabular}

$a_{n}=54$.

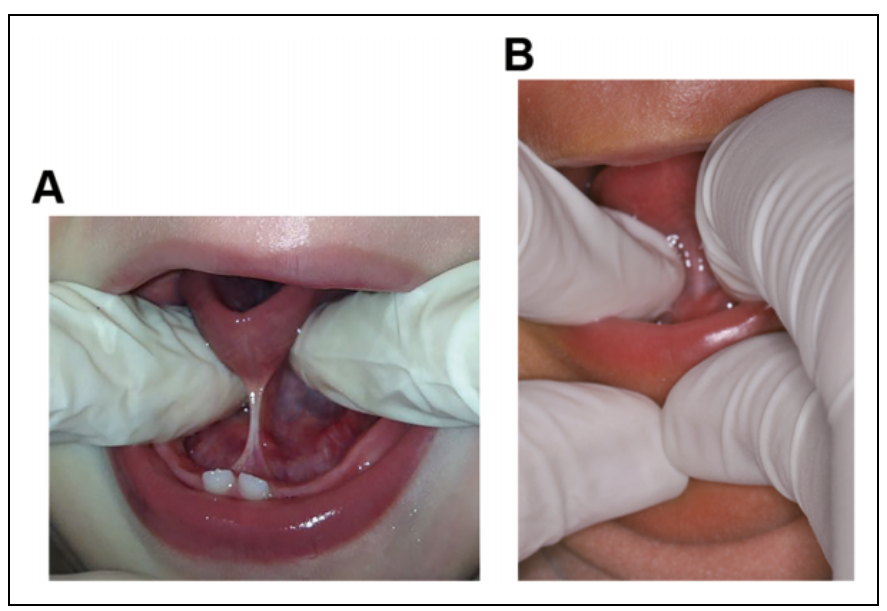

Figure 2. Most frequently observed lingual frenulum phenotypes in children with congenital Zika syndrome (CZS). A, A case in which lingual frenulum was visible with simple elevation maneuver. $B, A$ posteriorly positioned lingual frenulum without anatomy visibility (submucous), with tongue maneuver to a superior and posterior position.

\section{Discussion}

A wide variation within the anatomical spectrum of lingual frenulum has been reported in both healthy (Ghaheri et al., 2017) and syndromic children (Mintz et al., 2005). Recently, 


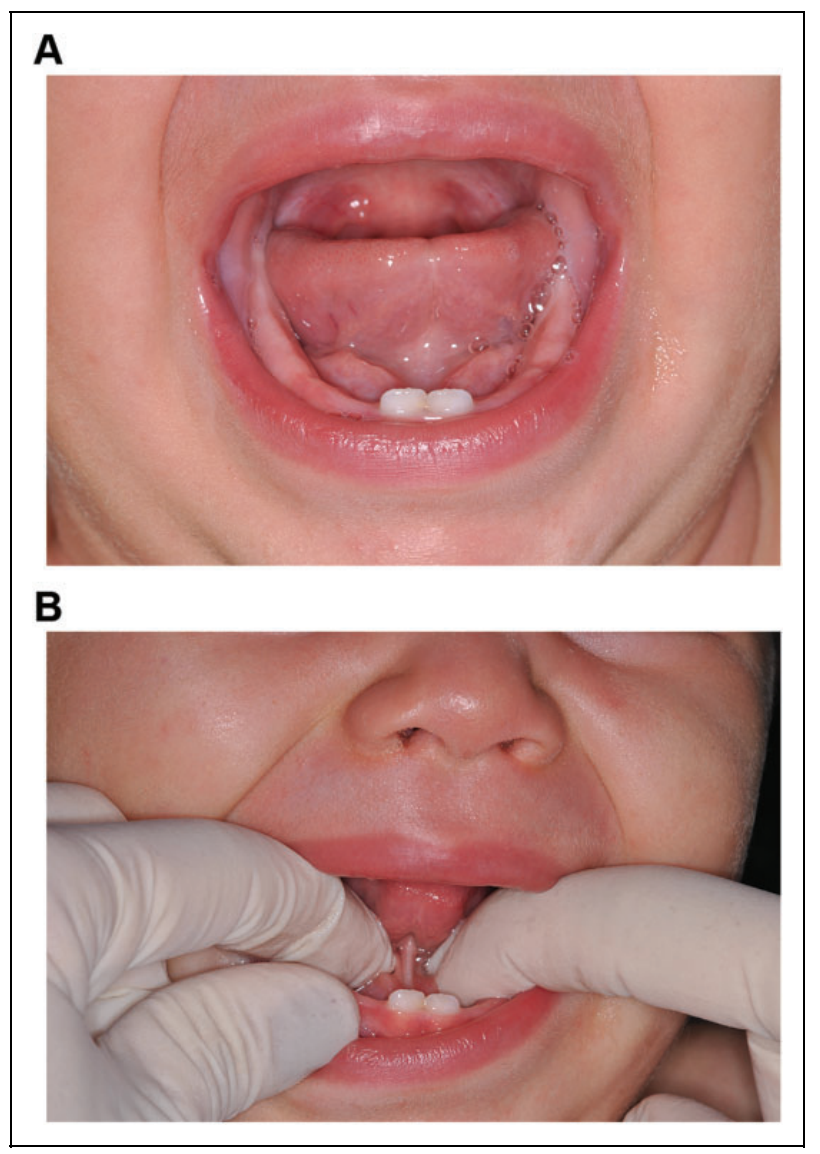

Figure 3. Posteriorly positioned lingual frenulum in I infant $(A / B)$, visible only after maneuver. $A$, The appearance of and absence of the lingual frenulum before maneuver is applied. B, Visibility of frenulum after maneuvering tongue to a superior and posterior position.
Del Campo et al. (2017) described the absence of lingual frenulum in 4 of 11 children affected by CZS. In the present study, many infants $(\mathrm{n}=20)$ affected by CZS presented a posteriorly positioned lingual frenulum, rather than absence of this small band of tissue. Furthermore, in 14 of 20 patients, the lingual frenulum was hidden behind a mucous curtain. Based on these findings, we suggest a change in the terminology proposed by Del Campo et al. (2017), with substitution of the term "absent" by the term "submucous" lingual frenulum. Previous work identified the absence of lingual frenulum in Ehlers-Danlos syndrome (OMIM 130010, OMIM 130020; De Felice et al., 2001). In these patients, the absence of labial or lingual frenulum may be considered an important means for the differential diagnosis of this condition (Machet et al., 2010). Ehlers-Danlos syndromes are a clinically and genetically heterogeneous group of inherited connective tissue disorders characterized by skin hyperextensibility, articular hypermobility, and tissue fragility (Beighton et al., 1969). Some clinical forms of Ehlers-Danlos syndrome are caused by mutations affecting collagen, an integral component of the oral mucous membrane, which may justify the absence of lingual frenulum in these patients. Nevertheless, CZS is the result of a prenatal infection of the developing brain structures by the Zika virus. A recent in vitro study showed that this virus also infects cranial neural crest cells (Bayless et al., 2016). The authors have suggested that since development of the brain and face integrates at various levels, signaling crosstalk between these structures appears to occur and may contribute to the embryopathies associated with CZS. In addition, previous knowledge that the tongue connective tissue and vasculature derive from cranial neural crest cells (Parada and Chai, 2015) has led us to propose that infection

Table 2. Association Between the Presence of Dysphagia and Posteriorly Positioned Lingual Frenulum Among Children With Congenital Zika Syndrome (CZS).

\begin{tabular}{|c|c|c|c|c|c|}
\hline Variable & & & \multicolumn{2}{|c|}{ Posterior_Frenulum } & Total \\
\hline \multirow[t]{10}{*}{ Dysphagia_Presence } & No & Count & 9 & I & 10 \\
\hline & & Expected count & 6.3 & 3.8 & 10.0 \\
\hline & & \% within Dysphagia_presence & $90.0 \%$ & $10.0 \%$ & $100.0 \%$ \\
\hline & & $\%$ within posterior_frenulum & $36.0 \%$ & $6.7 \%$ & $25.0 \%$ \\
\hline & & Expected count & 18.8 & 11.3 & 30.0 \\
\hline & & $\%$ within dysphagia_presence & $53.3 \%$ & $46.7 \%$ & $100.0 \%$ \\
\hline & & $\%$ within posterior_frenulum & $64.0 \%$ & $93.3 \%$ & $75.0 \%$ \\
\hline & & $\%$ of total & $40.0 \%$ & $35.0 \%$ & $75.0 \%$ \\
\hline & Total & Count & 25 & 15 & 40 \\
\hline & & Expected count & 25.0 & 15.0 & 40.0 \\
\hline
\end{tabular}

${ }^{a} n=40, n=40$, Chi-square $(p=.038)$ 
Table 3. Logistic Regression Model to Predict the Influence of Variables Over the Presence of Dysphagia.

\begin{tabular}{|c|c|c|c|c|c|c|}
\hline Variable & B & SE & Wald & $d f$ & Significance & $\operatorname{Exp}(B)$ \\
\hline Pyramidal/extrapyramidal signs & -2.439 & $1.34 \mathrm{I}$ & 3.306 & I & .069 & 0.087 \\
\hline Postural_control & 0.798 & 1.839 & 0.188 & I & .664 & 2.222 \\
\hline Global_muscular_tone & 0.556 & 1.882 & 0.087 & I & .767 & $\mathrm{I} .745$ \\
\hline Constant & -1.827 & 1.162 & 2.473 & I & .115 & 0.161 \\
\hline
\end{tabular}

Abbreviation: SE, standard error.

of these cells may explain the greater frequency of posterior and submucous lingual frenula observed in our study.

Although a posteriorly positioned lingual frenulum was a highly prevalent intraoral feature in the present study, the absence of lingual frenulum was not observed among children with CZS. In addition, a truly absent lingual frenulum is a very rare finding. Haham et al. (2014) reported 1 absent lingual frenulum among 200 healthy infants. We established a specific criteria for the differential diagnosis of submucous and absent lingual frenula and considered a lingual frenulum to be submucous when a discrete band or column of tissue located against the base of the tongue and covered by an overlapping curtain-like mucous membrane would protrude after elevating and retracting the tongue (specific maneuver), while an absent frenulum meant the total absence of a protruding band of tissue after elevating and retracting the tongue. In our experience, these diagnostic criteria are essential to correctly identify the lingual frenulum anatomy. Although the literature mentions the concept of an absent lingual frenulum, to our knowledge there is no available literature that defines or provides a proper criteria for the diagnosis of an absent frenulum. Furthermore, the few available studies that describe the absence of lingual frenulum do so based on visual inspection without providing detailed information on the assessment protocols used to determine frenulum absence (De Felice et al., 2001; Haham et al., 2014; Kamal et al., 2016).

There is a limited number of publications describing the anatomic characteristics of the lingual frenulum in a normal population of infants, and even fewer cohorts show the presence of a posteriorly positioned lingual frenulum covered by an overlapping mucosa, also known as submerse or submucous lingual frenulum (Chu and Bloom, 2009; Hong et al., 2010; Douglas, 2013). In addition, most of these studies present the bias of restricting their sample to patients who were referred for frenotomy/frenulectomy or have breastfeeding difficulties and differ in the criteria adopted for examination, using different forms of classification and/or description of the observed anatomic features. These studies show great variation in the prevalence of posteriorly positioned lingual frenulum. Martinelli et al. (2013) evaluated 100 healthy children and showed the presence of posterior lingual frenulum in $29 \%$ of their patients (Martinelli et al., 2013), whereas another study in a similarly healthy population $(n=166)$ reported a total of $0.6 \%(n=1)$ of posteriorly positioned lingual frenula (Marcione et al., 2016).
Hong et al. (2010) reported a case series of 341 infants who underwent outpatient frenotomy for breastfeeding difficulties secondary to anterior or posterior ankyloglossia and described a $6 \%$ prevalence of posterior ankyloglossia in their population $(n=19 / 341)$. Pransky et al. (2015) evaluated breastfeeding difficulties among infants and observed a $19 \%$ prevalence of posterior tongue-tie ( $\mathrm{n}=120 / 618$, Kotlow types III and IV). Haham et al. (2014) reported on the prevalence of lingual frenulum among 200 healthy newborn infants based on the Coryllos classification and showed a prevalence of $23 \%(n=47)$ of posteriorly positioned lingual frenulum. Dollberg et al. (2014) prospectively followed 244 infants who underwent frenotomy and reported a $32 \%$ prevalence of posteriorly positioned lingual frenulum $(n=78)$. Benoiton et al. (2016) $(46.5 \%, n=20 / 43)$ and Callahan et al. (2013) $(47 \%, \mathrm{n}=145 / 311)$ reported exceptionally high percentages of posterior lingual frenula. In these studies, samples consisted mainly of infants referred for frenotomy due to the presence of posterior ankyloglossia and/or upper lip tie (Benoiton et al., 2016) or infants with problematic ankyloglossia (Callahan et al., 2013), explaining the higher prevalence of posterior lingual frenula. In the present study, examination of infants with CZS demonstrated that $37 \%(\mathrm{n}=$ 20 ) of these patients had a posterior lingual frenulum and required elevation followed by the posterior maneuver of the tongue to adequately visualize the tissue. This prevalence was higher than previously reported results among healthy infants (Hong et al., 2010; Martinelli et al., 2013; Haham et al., 2014; Dollberg et al., 2014; Pransky et al., 2015; Marcione et al., 2016) and was only lower than the prevalence from studies that favored the recruitment of patients with this type of frenulum attachment (Benoiton et al., 2016; Callahan et al., 2013).

Presently, $70 \%(n=14)$ of infants with a posterior lingual frenulum had a submucous frenulum. It is important to consider that most studies do not differentiate a posteriorly positioned lingual frenulum from its submucous subtype (Hong et al., 2010; Martinelli et al., 2013; Haham et al., 2014; Dollberg et al., 2014; Pransky et al., 2015; Marcione et al., 2016). However, a submucous lingual frenulum if correctly identified may allow a more accurate understanding of its prevalence and impact within a healthy population of infants. Diagnosis of a submucous frenulum is often challenged by the need for proper tongue maneuver to provide visibility. In these situations, the lingual frenulum is frequently concealed behind a curtain-like mucous membrane (Chu and Bloom, 2009; Hong et al., 2010; 
Pradhan et al., 2012; Pradhan et al., 2013), thereby rendering a subtle appearance or no clinical sign of its presence by simple visual inspection. In our experience, appropriate tongue maneuver is essential for adequate visualization of an apparently absent lingual frenulum. Many studies do not describe the use of specific tongue maneuvers or devices to diagnose the presence/absence of a lingual frenulum, suggesting visual inspection or the use of simple elevation maneuver as a means for diagnosis. Along these lines, different devices and/or tools have been used to aid in the assessment of lingual frenulum insertion and anatomy, such as metal grooved elevator (Benoiton et al., 2016), groove director (Chu and Bloom, 2009), the Pradhan retraction device (Pradhan et al., 2013), the Hazelbaker assessment tool for lingual frenulum function (Hazelbaker, 2010), the Bristol tongue assessment tool (Ingram et al., 2015), Murphy maneuver (Pradhan et al., 2013), among others. Therefore, there are various methods that may be used to assess the presence/absence of ankyloglossia, and the lack of a standardized assessment tool may explain the insufficient agreement between studies. In the present work, we chose to use the tongue maneuver described by Martinelli et al. (2013). Our choice was justified by the ease of use by the multidisciplinary team and costeffectiveness of this technique, which dispenses tools or devices to access lingual frenulum insertion and anatomy.

Evaluation of global muscular tone, global posture, postural control, and cervical spine control allowed assessment of the degree of neurologic involvement of children with CZS. The results confirmed a high prevalence of hypertonia $(75.9 \%, \mathrm{n}=$ $41 / 54)$, disorganized global posture $(81.5 \%, n=44 / 54)$, inadequate cervical spine $(68.5 \%, \mathrm{n}=37 / 54)$, and postural control $(87 \%, \mathrm{n}=47 / 54)$. Most of these children are extremely irritable (with persistent and loud crying) and have a compromised cervical motor control (eg, they are unable to lift and hold the head and neck). These postural aspects of neurologic origin can significantly influence our ability to assess the lingual frenulum and the overall oral function. Hence, in these patients, tongue position tends to be hypotonic at rest, but once the child is stimulated and acquires a hypertonic posture, the tongue retracts, becomes tense, and elevates. In addition, these children have a strong clenching reflex. Our understanding is that these challenging aspects are of importance during intraoral evaluation and must be considered by professionals, particularly when assessing lingual frenulum anatomy. For intraoral assessment, we applied our maneuver protocol with the infant at rest, in their most relaxed position, on their mothers' or caretakers' arms to minimize the influence of these limiting factors and allow proper visualization of the lingual frenulum.

Children affected by CZS present different levels of dysphagia, and failure to thrive may become an issue in the most severe cases. In these instances, food intake is provided by alternative feeding methods (Svystun et al., 2017). Recently, Leal et al. (2017) assessed dysphagia in 9 infants with CZS and provided pertinent findings associated with this condition. In brief, all infants presented different levels of neurologic damage (such as global developmental delays, increased tonus of the limbs, pyramidal and extrapyramidal involvement) and major abnormalities in the oral phase, coupled with delayed initiation of the pharyngeal phase of swallowing. Abnormal movement of the tongue was detected in most infants and identified as a contributing factor to dysphagia. In the present work, we were able to collect data on the presence and degree of dysphagia of 40 infants. Only 10 infants of 40 did not show the presence of dysphagia. Most importantly, dysphagia demonstrated a significant association with the presence of a posteriorly positioned lingual frenulum, as well as with the presence of pyramidal/extrapyramidal signs of neurologic impairment, but was not confirmed in a binomial regression model probably due to our small sample size. Our results suggest that the presence of a posteriorly positioned lingual frenulum may be of clinical significance in oral function by affecting the child's ability to feed. However, the clinical significance of this type of morphology on speech can only be established once children are attempting to speak. All our patients are developmentally delayed and have not reached this developmental stage. It remains unclear the degree to which posterior ankyloglossia contributes to the development of feeding difficulties in infants. This is a controversial issue that has not yet been clarified in the literature. Although dysphagia is primarily a consequence of cortical and extrapyramidal neurologic damage, anatomic features such as a posteriorly positioned lingual frenulum may be among a broad array of contributory issues that must be considered during clinical assessment. A significant number of patients in our study sample present oral phase dysphagia, and the most severe cases are being treated with alternative feeding methods. Interestingly, most of these infants are able to breastfeed, showing more difficulty with bottle or spoon feeding.

\section{Conclusions}

In conclusion, the present findings suggest that the absence of lingual frenulum is not observed among infants with CZS; nevertheless, many of these patients present a posterior lingual frenulum that without specific maneuver may be misinterpreted as an absent frenulum. The presence of posteriorly positioned lingual frenulum is associated with dysphagia in this population. Further prospective studies in a larger population of children with CZS to establish the level of interference of a posteriorly positioned lingual frenulum on tongue function and its relation to normal swallowing are warranted.

\section{Declaration of Conflicting Interests}

The author(s) declared no potential conflicts of interest with respect to the research, authorship, and/or publication of this article.

\section{Funding}

The author(s) received no financial support for the research, authorship, and/or publication of this article.

\section{References}

Bayless NL, Greenberg RS, Swigut T, Wysocka J, Blish CA. Zika virus infection induces cranial neural crest cells to produce 
cytokines at levels detrimental for neurogenesis. Cell Host Microbe. 2016;20(4):423-428.

Beighton PH, Price A, Lord J, Dickson ER. Variants of the Ehlers-Danlos syndrome: clinical, biochemical, haematological, and chromosomal features of 100 patients. Ann Rheum Dis. 1969;28(3):228-245.

Benoiton L, Morgan M, Baguley K. Management of posterior ankyloglossia and upper lip ties in a tertiary otolaryngology outpatient clinic. Int J Pediatr Otorhinolaryngol. 2016;88:13-16.

Campos GS, Bandeira AC, Sardi SI. Zika virus outbreak, Bahia, Brazil. Emerg Infect Dis. 2015;21:1885-1886.

Cavalcanti LPG, Ribeiro EM, Pessoa ALS, et al. Microcephaly in infants, Ceará State, Brazil, 2015-2016. Rev Med UFC. 2017;57:52-57.

Chu MW, Bloom DC. Posterior ankyloglossia: a case report. Int $J$ Pediatr Otorhinolaryngol. 2009;73:881-883.

de Fatima Vasco Aragao M, Van der Linden V, Brainer-Lima AM, et al. Clinical features and neuroimaging (CT and MRI) findings in presumed Zika virus related congenital infection and microcephaly: retrospective case series study. BMJ. 2016;353:i3182.

De Felice C, Toti P, Di Maggio G, Parrini S, Bagnoli F. Absence of inferior labial or lingual frenula in Ehlers-Danlos syndrome. Lancet. 2001;357(9267):1500-1502.

Del Campo M, Feitosa IM, Ribeiro EM, et al. Zika Embryopathy Task Force-Brazilian Society of Medical Genetics ZETF-SBGM. The phenotypic spectrum of congenital Zika syndrome. Am J Med Genet A. 2017;173(4):841-857.

Dollberg S, Marom R, Botzer E. Lingual frenotomy for breastfeeding difficulties: a prospective follow-up study. Breastfeeding Med. 2014;9(6):286-289.

Douglas PS. Rethinking “posterior” tongue-tie. Breastfeed Med. 2013; 8:503-506.

Fehrenbach MJ, Herring SW. Illustrated Anatomy of the Head and Neck. St Louis, MO: WB Saunders Company; 2002.

Ghaheri BA, Cole M, Fausel SC, Chuop M, Mace JC. Breastfeeding improvement following tongue-tie and lip-tie release: a prospective cohort study. Laryngoscope. 2017;127(5):1217-1223.

Haham A, Marom R, Mangel L, Botzer E, Dollberg S. Prevalence of breastfeeding difficulties in newborns with a lingual frenulum: a prospective cohort series. Breastfeeding Med. 2014;9(9): 438-441.

Hazelbaker AK. Tongue-Tie Morphogenesis, Impact, Assessment and Treatment. Columbus, OH: Aidan \& Eva Press; 2010.

Heukelbach J, Alencar CH, Kelvin AA, Oliveira WK, Cavalcanti LPG. Zika virus outbreak in Brazil. J Infect Dev Ctries. 2016; 10(2):116-120.

Hong P, Lago D, Seargeant J, Pellman L, Magit AE, Pransky SM. Defining ankyloglossia: a case series of anterior and posterior tongue ties. Int J Pediatr Otorhinolaryngol. 2010;74(9):1003-1006.

Ingram J, Johnson D, Copeland M, Churchill C, Taylor H, Emond A. The development of a tongue assessment tool to assist with tonguetie identification. Arch Dis Child Fetal Neonatal Ed. 2015;100(4): F344-F348.

Kamal K, Bansal T, Saini S, Vashisht G. Fixing endotracheal tubes in patients with an absent lingual frenulum. Anaesthesia. 2016;71(4): 478-479.

Knox I. Tongue tie and frenotomy in the breastfeeding newborn. NeoReviews. 2010;11(9):513-519.
Leal MC, van der Linden V, Bezerra TP, Borges ACG, Antunes MMC, Brandt KG, Moura CX, Rodrigues LC, Ximenes CR. Characteristics of dysphagia in infants with microcephaly caused by congenital Zika virus infection, Brazil, 2015. Emerg Infect Dis. 2017;23(8):1253-1259.

Machet L, Hüttenberger B, Georgesco G, et al. Absence of inferior labial and lingual frenula in Ehlers-Danlos syndrome. A minor diagnostic criterion in French patients. Am J Clin Dermatol. 2010;11(4):269-273.

Marchesan IQ, Oliveira LR, Martinelli RLC. Frênulo da línguaControvérsias e Evidências. In: Marchesan IQ, Silva HJ, Tomé MC. Tratado das Especialidades em Fonoaudiologia. São Paulo, Brazil: Roca; 2014:283-301.

Marcione ESS, Coelho FG, Souza CB, França ECL. Classificação anatômica do frênulo lingual de bebês. Revista CEFAC. 2016; 18(5):1042-1049.

Martinelli RLC, Marchesan IQ, Berretin-Felix G. Protocol for infants: relationship between anatomic and functional aspects. Rev CEFAC. 2013;15:599-609.

Mintz SM, Siegel MA, Seider PJ. An overview of oral frena and their association with multiple syndromic and nonsyndromic conditions. Oral Surg Oral Med Oral Pathol Oral Radiol Endod. 2005;99(3): 321-324.

Moore CA, Staples JE, Dobyns WB, et al. Characterizing the pattern of anomalies in congenital Zika syndrome for pediatric clinicians. JAMA Pediatr. 2017;171(3):288-295.

O'Callahan C, Macary S, Clemente S. The effects of office-based frenotomy for anterior and posterior ankyloglossia on breastfeeding. Int J Pediatr Otorhinolaryngol. 2013;77(5):827-832.

Parada C, Chai Y. Mandible and tongue development. Curr Top Dev Biol. 2015;115: 31-58.

Pradhan S, Yasmin E, Mehta A. Management of posterior ankyloglossia using the Er, Cr: YSGC Laser. Int J Laser Dentistry. 2012;2:41-46.

Pradhan S, Yasmin E, Shan AM. Ankyloglossia: the diagnostic dilemma. Int J Laser Dent. 2013;3:19-23.

Pransky SM, Lago D, Hong P. Breastfeeding difficulties and oral cavity anomalies: the influence of posterior ankyloglossia and upper-lip ties. Int J Pediatr Otorhinolaryngol. 2015;79(10):1714-1717.

Schuler-Faccini L, Ribeiro EM, Feitosa IM, Horovitz DD, Cavalcanti DP, Pessoa A, Doriqui MJ, Neri JI, Neto JM, Wanderley HY, et al. Possible association between Zika virus infection and microcephaly-Brazil, 2015. Morb Mortal Wkly Rep. 2016; 65(3):59-62.

Svystun O, Johannsen W, Persad R, Turner JM, Majaesic C, El-Hakim $\mathrm{H}$. Dysphagia in healthy children: characteristics and management of a consecutive cohort at a tertiary centre. Int J Pediatr Otorhinolaryngol. 2017;99:54-59.

Van der Linden V, Pessoa A, Dobyns W, Barkovich AJ, Júnior HVDL. Description of 13 infants born during October 2015January 2016 with congenital Zika virus infection without microcephaly at birth-Brazil. MMWR Morb Mortal Wkly Rep. 2016; 65(47);1343-1348

Ventura CV, Maia M, Bravo Filho V, Gois AL, Belfort R Jr. Zika virus in Brazil and macular atrophy in a child with microcephaly. Lancet. 2016;387(10015):228. 